\title{
Effective Treatment of Atrophic and Icepick Acne Scars Using Deep Non-Ablative Radiofrequency and Multisource Fractional RF Skin Resurfacing
}

\author{
Monica Elman ${ }^{1}$, Ido Frank ${ }^{2}$, Hila Cohen-Froman ${ }^{2}$, Yoram Harth ${ }^{2,3}$ \\ ${ }^{1}$ Beit Harofim Medical Center, Holon, Israel; ${ }^{2}$ EndyMed Medical Ltd., Cesarea, Israel; ${ }^{3} \mathrm{OR}$ Aesthetic Clinic, Herzlya, Israel. \\ Email: skin58@gmail.com
}

Received August 19 $9^{\text {th }}, 2012$; revised September 20 $0^{\text {th }}, 2012$; accepted September $30^{\text {th }}, 2012$

\begin{abstract}
Background and Objectives: Effective treatment for atrophic (depressed) and icepick acne scars requires treatment of both epidermis - for roughness, texture and hyperpigmentation, and the dermis - for collagen remodeling. All first generation radiofrequency systems allow nonablative RF treatment while a few others allow simple bipolar Fractional RF skin resurfacing. The FDA cleared multisource radiofrequency therapy system (EndyMed PRO, EndyMed Ltd., Cesarea, Israel) allows, for the first time, phase controlled multisource RF for both deep (up to $11 \mathrm{~mm}$ ) non ablative RF and fractional RF skin resurfacing on the same treatment device. Study Design/Materials and Methods: Ten subjects with atrophic acne scars were enrolled in the study. Patients were photographed using standardized methods. In each treatment session, each patient received a full face 3DEEP non ablative skin tightening treatment followed by a Fractional skin resurfacing treatment. The treatment sessions were repeated once a month to a total of up to 4 treatments. Results: All subjects experienced mild-moderate edema and erythema as an immediate response to treatment. Edema resolved after up to three hours post treatment and erythema lasted up to 2 days. Micro ablative crusts were formed $1-2$ days post treatments and lasted up to 5 days on facial areas. Patients' photographs - before each treatment session and 1 and 3 months after the last session - were graded according to the accepted Cosmetic Improvement Scale. All patients obtained significant skin improvement. Seventy percent of patients had 50\% - 75\% improvement one month after the third treatment session while the other thirty percent experienced a $25 \%-50 \%$. Discussion and Conclusions: The presented results describe for the first time a new treatment system $\left(\right.$ EndyMed $\mathrm{PRO}^{\mathrm{TM}}$ ) that allows both deep non ablative RF delivery and Fractional skin resurfacing on the same treatment platform. This work shows the synergy of combining these two applications for the improvement of acne scars, as well as for some secondary possible pigmentation and vascular improvement.
\end{abstract}

Keywords: Fractional Radio Frequency; Skin Resurfacing; Acne Scars

\section{Introduction}

Effective treatment for atrophic depressed acne scars requires treatment of both epidermis - for epidermal roughness, texture and hyperpigmentation and dermis-for collagen remodeling [1-3]. Many treatment modalities can improve color and texture. Nevertheless, building new collagen is dependent on the amount and volume of heat delivered to the deep dermis. The improvement offered by fractional lasers or fractional bipolar RF systems is usually limited, due to the small volume of dermal heat produced by these devices.

A new multisource RF, FDA cleared therapy system (EndyMed PRO, EndyMed Ltd., Cesarea, Israel) allows for the first time phase controlled multisource RF for both deep (up to $11 \mathrm{~mm}$ ) non ablative RF together with multisource fractional RF skin resurfacing on the same treatment device.

In the current study, we used a combined protocol that included two complementary treatments in one session-a full face non ablative skin tightening, followed by Fractional Skin Resurfacing (FSR). Both treatments were performed using the EndyMed PRO multisource radiofrequency system. The EndyMed PRO RF System is an FDA cleared computerized system that employs up to six radio frequency generators at the same time, creating few simultaneous electric fields in the same phase, that force the energy to penetrate into the deep dermis, causing a non-ablative deep dermal heating effect that results in painless skin tightening. In another handpiece, the multisource RF is used to form micro ablative fractional skin resurfacing with simultaneous volumetric 
heating of the upper dermis (Figures $\mathbf{1}$ and 2).

The non-ablative skin tightening application was applied first, creating a continuous deep volumetric dermal heating for stimulation of collagen remodeling, followed by the FSR (Fractional skin Resurfacing) application that reinforced the volumetric heating on the upper dermis and added micro-ablative resurfacing in up to $10 \%$ of the affected area.

\section{Methods}

Ten subjects with atrophic acne scars were enrolled in the study. Patients were photographed using standardized methods. In each treatment session, each patient received a full face 3DEEP non ablative skin tightening treatment followed, 45 minutes after, by a Fractional Skin Resurfacing treatment. A topical anesthetic (EMLA) was applied after the non ablative treatment to prepare skin for the fractional treatment. The treatment sessions were repeated at a minimum interval of once a month for a total of up to 4 treatments.

Before starting the non ablative procedure the skin was cleaned with $70 \%$ alcoholic gauze in order to remove make up or other lotions. The patients had up to 8 minute treatment in each area in which skin surface temperature was maintained at $40-42$ degrees Celsius. Before the FSR treatment the residual EMLA was removed gently with gauze containing $70 \%$ alcohol. During the treatment,

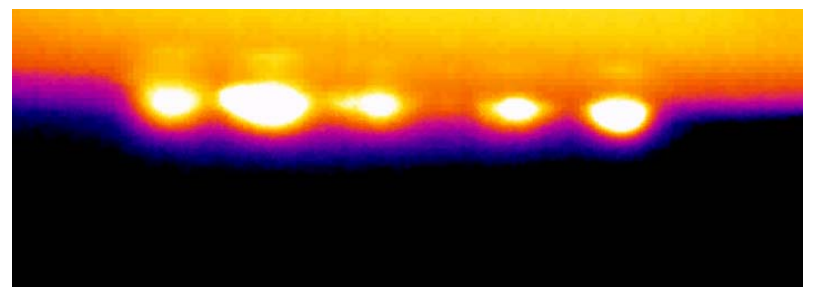

Figure 1. Thermal imaging of the Ablative phase of the Fractional Skin Resurfacing application pulse of EndyMed PRO multi-source RF system (EndyMed Ltd., Cesarea, Israel), showing the creation of the micro-ablative effect (ThermaCAM SC 640).

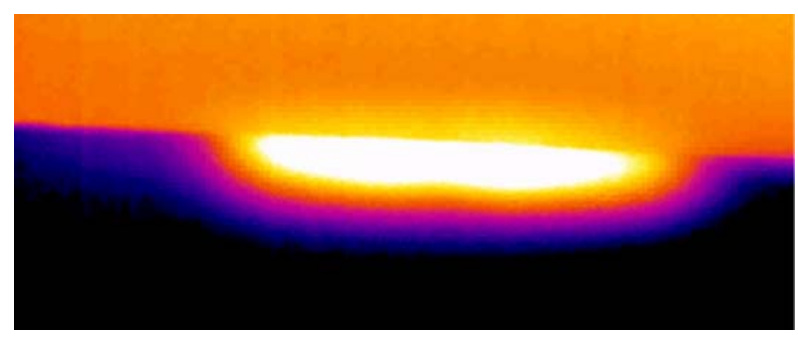

Figure 2. Thermal imaging of the volumetric phase of the Fractional Skin Resurfacing application pulse of EndyMed PRO multi-source RF system (EndyMed Ltd., Cesarea, Israel), showing significant volumetric heating (ThermaCAM SC 640). the treatment tip was placed on the skin and a short $(<1$ sec.) RF pulse of energy was delivered. Then, the tip was placed next to the first area and another RF pulse was delivered. This scenario was repeated until the whole treatment area was covered. In each treatment session, the fractional treatment was repeated 1 - 3 times over the whole scar area.

Treated areas were visually assessed for skin responses, including edema, erythema, hypopigmentation, hyperpigmentation, and textural changes following the treatment.

The use of a moisturizing cream (Ortho Dermatologics Topical Emulsion, Biafine) was recommended post treatment.

Patients' photographs were graded according to scales accepted by board certified dermatologists. Dedicated questionnaires were used for both doctor and patients in order to grade different parameters such as efficacy, safety, comfort of treatment, in addition to subjective satisfaction of the patients.

\section{Results}

The patients, nine women, one man, ages 21 - 70 years (average 37.2), were enrolled in the study after meeting all inclusion/exclusion criteria and providing signed informed consent forms.

All patients completed three to four treatment sessions, except for two patients who had only 2 sessions, due to unrelated personal reasons. Mild to moderate erythema and mild edema were noted following the non ablative Skin Tightening procedure. By the time the Fractional RF procedure started, the erythema was faded. Following the FSR treatment mild to moderate erythema and edema were noted. Erythema lasted up to one day. Micro crusts were formed one to two days following the treatment. These crusts started to peel off 3 - 4 days post treatment and totally resolved within $4-5$ days following treatment. Post treatment instruction included gentle cleaning of the face during the first days after the treatment, and a four week intense regimen of high potency sunblock. The male patient was advised not to shave for one week. No unexpected adverse effects were detected or reported. There were no incidences of infections, scarring, hyper/ hypopigmentation, or any other serious complications.

\subsection{Physician Evaluation}

$70 \%$ of the patient showed $51 \%-75 \%$ improvement in skin texture, roughness and acne scar appearance, while the other $30 \%$ of the patients showed $26 \%-50 \%$ improvement (Figure 3). When evaluated 3 months after the last session, all patients showed significant improvement in skin texture and scar appearance (Figures 4-6). 


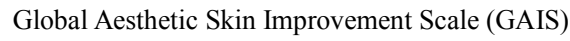

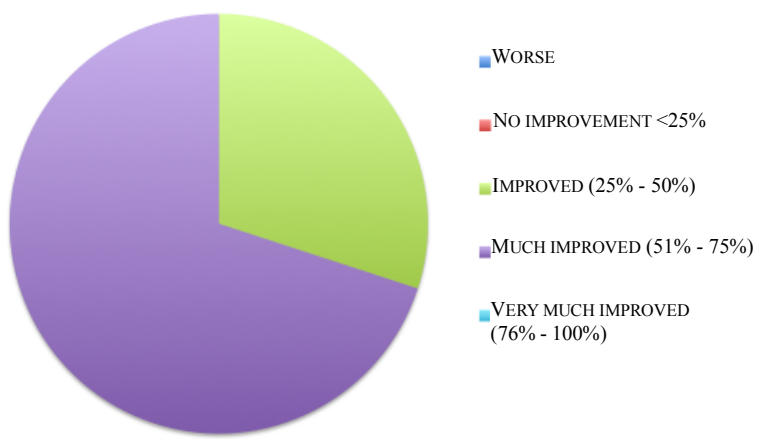

Figure 3. The overall improvement in skin appearance, 3 months after the last session.

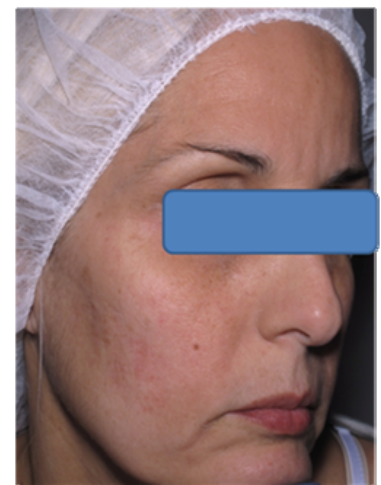

Baseline

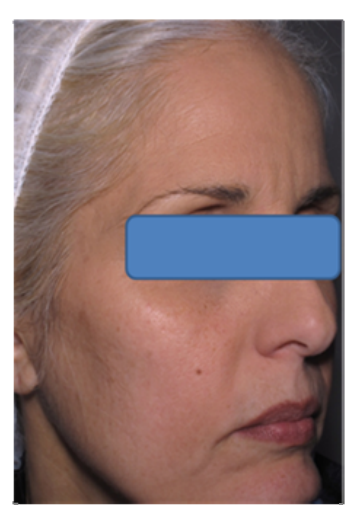

After 1 Combined treatment
Figure 4. Significant improvement in skin texture and reduction of acne scars appearance after one combined session (Left-Baseline, Right-After one combined session). Parameters; 3 - 4 Watts, 30 - 40 msec. Facial Skin Tightening handpiece and Fractional Skin Resurfacing handpiece of the EndyMed PRO multi-source RF system (EndyMed Ltd., Cesarea, Israel.) Photo: Dr. Monica Elman, Holon, Israel.

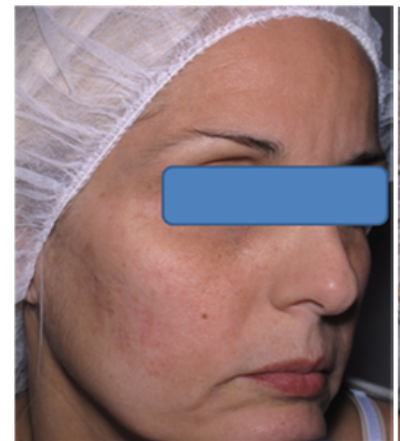

Baseline

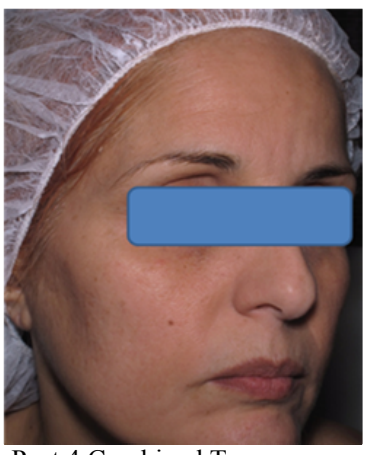

Post 4 Combined Tx
Figure 5. Figure 4 demonstrates further improvement in skin texture and reduction of acne scars appearance after four combined Sessions (Left-Baseline, Right-After four combined sessions). Parameters; 3 - 4 Watts, 30 - 40 msec. Facial Skin Tightening handpiece and Fractional Skin Resurfacing handpiece of the EndyMed PRO multi-source RF system (EndyMed Ltd., Cesarea, Israel.) Photo: Dr. Monica Elman, Holon, Israel.

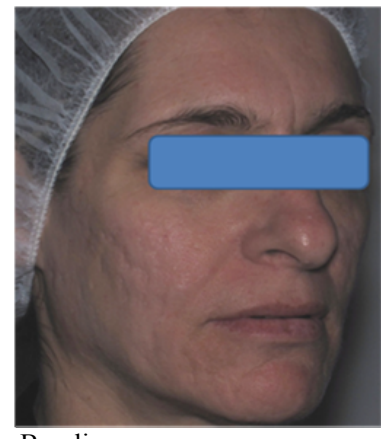

Baseline

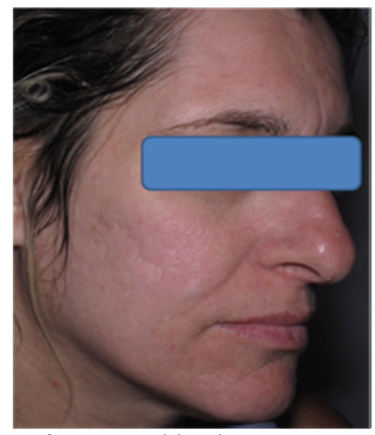

After 1 Combined treatment
Figure 6. Significant improvement in skin texture and reduction of acne scar appearance after one combined session. Parameters; 3 - 4 Watts, 30 - 40 msec. Facial Skin Tightening handpiece and Fractional Skin Resurfacing handpiece of the EndyMed PRO multi-source RF system (EndyMed Ltd., Cesarea, Israel.) Photo: Dr. Monica Elman, Holon, Israel.

\subsection{Reduction of Pigmentation and Small Blood Vessels Congestion}

Improvement in skin pigmentation and appearance of capillaries was not in the initial scope of this study. However, patients' photos were taken using a multi-filter camera (Reveal Imager, Canfield Imaging Systems, NJ, USA) that enables filtering brown and red spots.

Applying these filters and evaluation of the baseline photos compared to those of the following treatments revealed interesting data-some of the patients demonstrated a mild to moderate improvement in the amount of red and brown spots, meaning decrease in skin pigmentation and superficial blood vessels (Figures 7 and 8). This result may be attributed to the intense bulk heating caused by the two applications and mainly by the FSR application.

\subsection{Patients' Experience}

Patients' experience was evaluated using multiple choice questionnaires.

When asked about their subjective opinion about the cosmetic change they experienced, $30 \%$ reported $51 \%$ $75 \%$ improvement at the end of the last session. When asked the same question 3 months post the last treatment, $50 \%$ of the patients reported $51 \%-75 \%$ improvement (Figure 9).

When asked "Did the treatment meet your expectations?" At the end of the last session, 40\% responded "yes" while $60 \%$ responded "yes to some extent". When asked the same question one month and 3 months after the end of treatment, 50\% answered "yes" (Figure 10).

When asked at the end of three treatments "Would you recommend the treatment to your colleagues and friends?" $80 \%$ of patients answered definitely yes and 

Multisource Fractional RF Skin Resurfacing
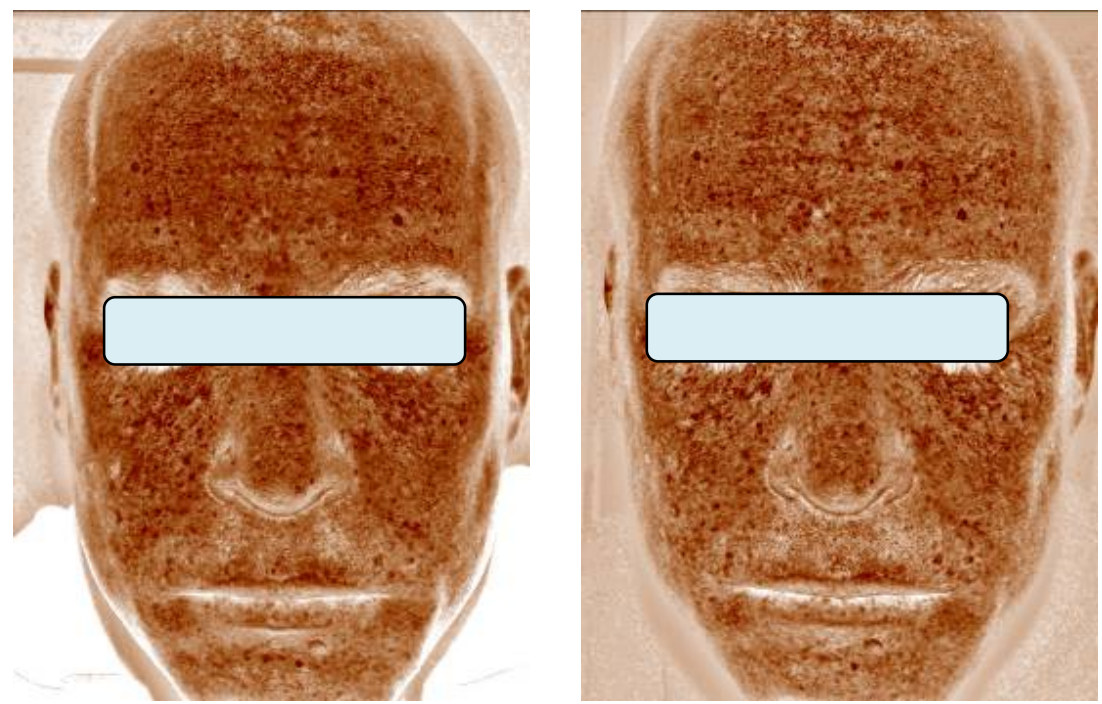

Figure 7. Mild reduction in the level of skin pigmentation. Left-Baseline; Right—Post one combined session.
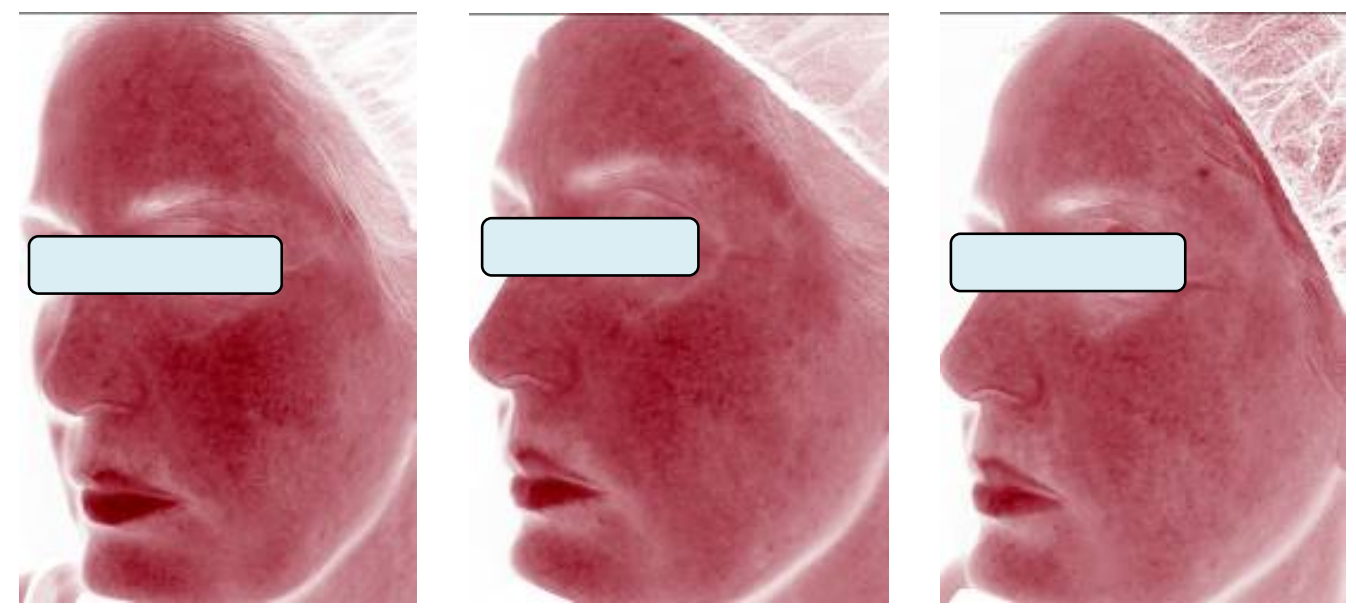

Figure 8. Reduction of redness and capillary congestion. Left-Baseline; Middle-Post two combined sessions; Right-3 months after three combined sessions.

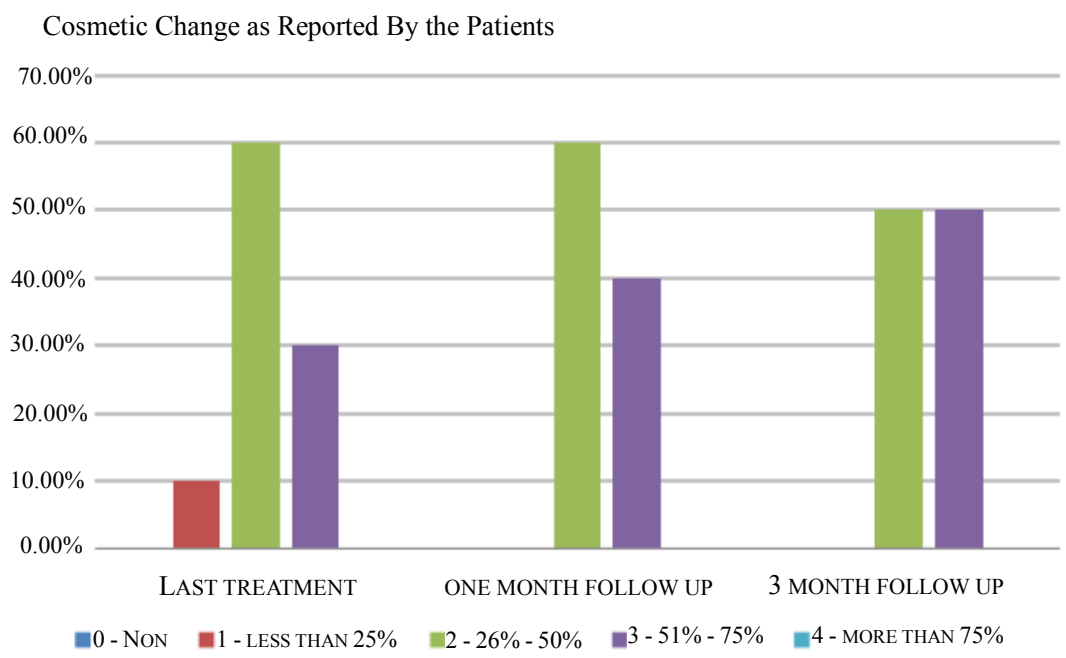

Figure 9. Patients opinion regarding their cosmetic improvement ( $\mathrm{Y}$ axis $=$ Percent of the patient group). 


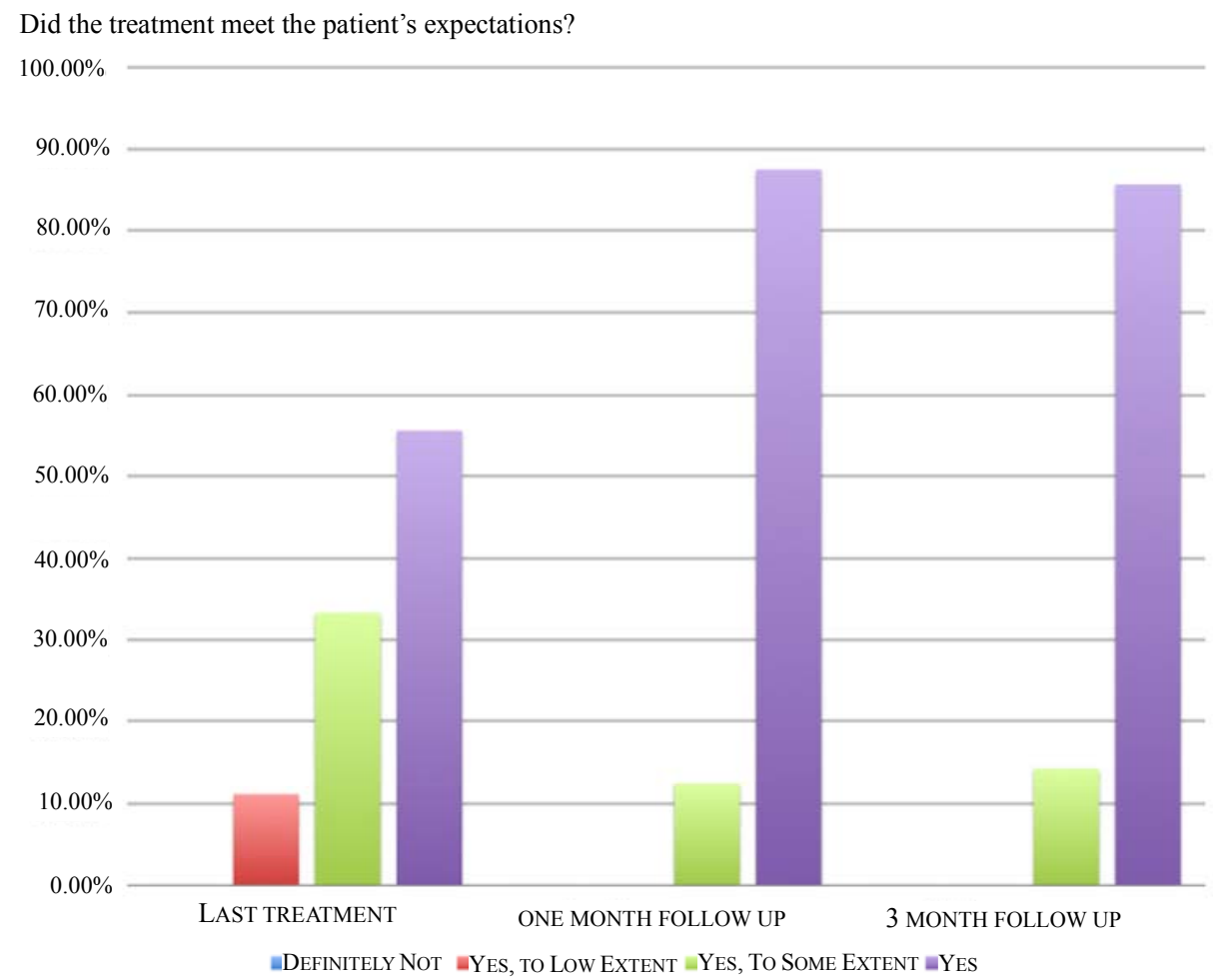

Figure 10. Patients were asked whether the treatment fulfilled their expectations; after completing three treatment sessions, and one and 3 months after the last treatment session ( $\mathrm{Y}$ axis $=$ percent of the patient group).

$20 \%$ answered yes. When asked the same question 3 months after the end of treatment all patients $(90 \%)$ answered definitely yes.

\section{Discussion and Conclusions}

Multisource radiofrequency (EndyMed PRO) is one of the leading technologies for body and face non ablative skin tightening. In this present report we describe, for the first time, clinical data from a study using a unique combination of Multisource RF Skin Tightening application together with Fractional RF applicator on the same platform. This RF based Multisource Fractional RF applicator enables, together with the non ablative skin tightening, a combination of RF microablation and volumetric deep dermal heating for the simultaneous epidermal and dermal skin rejuvenation effect [4-8].

The intrinsic disadvantage of current fractional laser systems is the low volume of dermal heating (narrow laser beam) and the inability to differentiate between epidermal ablation and dermal heating. Fractional lasers must be used at a higher laser power in order to increase volumetric heating in the dermis. This will significantly increase epidermal ablation downtime and possible side effects such as prolonged erythema and post inflammatory hyperpigmentation. Decreasing laser power will provide good epidermal results with little or no dermal effect [8].

Fractional resurfacing using radiofrequency results in dry microablation, thus downtime is shorter and the risk for side effects such as infection are minimal as compared to laser resurfacing methods that result in open wounds. Clinically, the affected areas are erythematous and mildly edematous after treatment, but resolve within few hours. This rapid healing is likely related to the persistence of healthy unaffected tissue that remains between the ablated pulses after ablative fractional resurfacing [8-11].

Laboratory thermal studies using EndyMed's Multisource Fractional handpiece on Agar have shown volumetric heating of the dermis up to $2.8 \mathrm{~mm}$, with a concomitant epidermal ablation with a contact diameter of 300 micron and a depth of $100-150$, which is optimal for fractional epidermal skin resurfacing. Animal studies have shown the formation of micro crusts $1-2$ days after treatment with full recovery of the epidermis in $5-7$ days.

Sadick et al. have used the EndyMed PRO on thirty patients treating wrinkles on the face and acne scars. They reported clinical improvement in skin texture in patients with both wrinkles and acne scars. They also noted that the incidence of complications was much lower than that seen following traditional ablative laser 
resurfacing [12-14].

The treatment was well accepted by the patients with minimal or no pain after the application of a $5 \%$ topical anesthetic cream (Emla). There was no need for post therapy treatment and patients were able to return to work with makeup as soon as one hour after therapy. Micro crusts developed 24 - 48 hours after therapy and resolved 4 - 5 days after therapy.

Beneficial epidermal effects were noted starting one week after therapy and included significantly improved brightness of the skin, smoothening of the skin and some secondary decrease in pigmentary changes (post acne and senile lentigines). Four to six weeks after treatment dermal changes were noted including significant improvement in skin texture, reduction of wrinkles and skin laxity.

Based on the above results, we believe the new EndyMed PRO combined treatment which combines non invasive skin tightening followed by multisource Fractional skin resurfacing procedure, provides an exciting new option for effective multilayer skin rejuvenation and treatment of skin laxity with minimal discomfort and downtime. We found that this specific protocol delivers better results than traditional lasers for acne scars and superficial skin imperfections and about the same results for the treatment of facial wrinkles.

\section{REFERENCES}

[1] A. M. Chapas, L. Brightman, S. Sukal, et al., "Successful Treatment of Cuneiform Scarring with $\mathrm{CO}_{2}$ Ablative Fractional Resurfacing," Journal of Lasers in Surgery and Medicine, Vol. 40, No. 6, 2008, pp. 381-386. doi:10.1002/1sm.20659

[2] S. E. Walgrave, A. E. Ortiz, H. T. MacFalls, et al., "Evaluation of a Novel Fractional Resurfacing Device for Treatment of Acne Scarring," Journal of Lasers in Surgery and Medicine, Vol. 41, No. 2, 2009, pp. 122-127. doi:10.1002/1sm.20725

[3] A. E. Ortiz, A. M. Tremaine and C. B. Zachary, "LongTerm Efficacy of a Fractional Resurfacing Device," Journal of Lasers in Surgery and Medicine, Vol. 42, No. 2, 2010, pp. 168-170. doi:10.1002/1sm.20885

[4] M. Elman and Y. Harth, "Novel Multi-Source PhaseControlled Radiofrequency Technology for Non-Ablative and Micro-Ablative Treatment of Wrinkles, Lax Skin and Acne Scars," Journal of Laser Therapy, Vol. 20, No. 2, 2011, pp. 139-144. doi:10.5978/islsm.20.139

[5] Y. Harth and D. Lischinsky, "A Novel Method for Real-
Time Skin Impedance Measurement during Radiofrequency Skin Tightening Treatments," Journal of Cosmetic Dermatology, Vol. 10, No. 1, 2011, pp. 24-29. doi:10.1111/j.1473-2165.2010.00535.x

[6] J. R. de la Torre, J. Moreno-Moraga, E. Muñoz, et al., "Multisource, Phase-Controlled Radiofrequency for Treatment of Skin Laxity: Correlation between Clinical and in Vivo Confocal Microscopy Results and Real-Time Thermal Changes," Journal of Clinical and Aesthetic Dermatology, Vol. 4, No. 1, 2011, pp. 28-35.

[7] M. Elman, I. Vider, Y. Harth, et al., "Non-Invasive Therapy of Wrinkles, Lax Skin Using a Novel Multi-Source Phase Controlled Radiofrequency System," Journal of Cosmetic and Laser Therapy, Vol. 12, No. 2, 2010, pp. 81-86. doi:10.3109/14764171003706133

[8] B. M. Hantash, V. P. Bedi, K. F. Chan, et al., "Ex Vivo Histological Characterization of a Novel Ablative Fractional Resurfacing Device," Journal of Lasers in Surgery and Medicine, Vol. 39, No. 2, 2007, pp. 87-95. doi:10.1002/1sm.20405

[9] N. S. Sadick, M. Sato, D. Palmisano, et al., "In Vivo Animal Histology and Clinical Evaluation of Multisource Fractional Radiofrequency Skin Resurfacing (FSR) Applicator," Journal of Cosmetic and Laser Therapy, Vol. 13, No. 5, 2011, pp. 204-209. doi:10.3109/14764172.2011.606467

[10] B. M. Hantash, V. P. Bedi, B. Kapadia, et al., "In Vivo Histological Evaluation of a Novel Ablative Fractional Resurfacing Device," Journal of Lasers in Surgery and Medicine, Vol. 39, No. 2, 2007, pp. 96-107. doi: $10.1002 / 1 \mathrm{sm} .20468$

[11] L. Brightman, M. P. Goldman and A. F. Taub, "Sublative Rejuvenation: Experience with a New Fractional Radiofrequency System for Skin Rejuvenation and Repair," Journal of Drugs in Dermatology, Vol. 8, No. 11, 2009, pp. 9-13.

[12] S. Walia and T. S. Alster, "Prolonged Clinical and Histologic Effects from $\mathrm{CO}_{2}$ Laser Resurfacing of Atrophic Acne Scars," Journal of Dermatologic Surgery, Vol. 25, No. 12, 1999, pp. 926-930. doi:10.1046/j.1524-4725.1999.99115.x

[13] C. A. Nanni and T. S. Alster, "Complications of Carbon Dioxide Laser Resurfacing. An Evaluation of $500 \mathrm{~Pa}-$ tients," Journal of Dermatologic Surgery, Vol. 24, No. 3, 1998, pp. 315-320.

[14] E. L. Tanzi and T. S. Alster, "Single-Pass Carbon Dioxide versus Multiple-Pass Er: YAG Laser Skin Resurfacing: A Comparison of Postoperative Wound Healing and Side-Effect Rates," Journal of Dermatologic Surgery, Vol. 29, No. 1, 2003, pp. 80-84. doi:10.1046/j.1524-4725.2003.29012.x 